\title{
Effective nonlinear model of resonant tunneling nanostructures
}

\author{
Enrique Diez and Angel Sánchez \\ Departamento de Matemáticas, Universidad Carlos III de Madrid, C./ Butarque 15, E-28911 \\ Leganés, Madrid, Spain \\ Francisco Domínguez-Adame \\ Departamento de Física de Materiales, Facultad de Físicas, Universidad Complutense, E-28040 \\ Madrid, Spain
}

\begin{abstract}
We introduce a model of a nonlinear double-barrier structure, to describe in a simple way the effects of electron-electron scattering while remaining analytically tractable. The model is based on a generalized effective-mass equation where a nonlinear local field interaction is introduced to account for those inelastic scattering phenomena. Resonance peaks seen in the transmission coefficient spectra for the linear case appear shifted to higher energies depending on the magnitude of the nonlinear coupling. Our results are in good agreement with self-consistent solutions of the Schrödinger and Poisson equations. The calculation procedure is seen to be very fast, which makes our technique a good candidate for rapid approximate analysis of these structures.
\end{abstract}


Resonant tunneling (RT) through double-barrier structures (DBS) make these systems very promising candidates for a new generation of ultra-high speed electronic devices. For instance, a GaAs- $\mathrm{Ga}_{1-x} \mathrm{Al}_{x}$ As DBS operating at $\mathrm{THz}$ frequencies has been reported in the literature. [1] The basic reason for RT to arise in DBS is a quantum phenomenon whose fundamental characteristics are by now well understood: There exists a dramatic increase of the electron transmittivity whenever the energy of the incident electron is close to one of the unoccupied quasi-bound-states inside the well. [2] In practice, a bias voltage is applied to shift the energy of this quasi-bound-state of nonzero width so that its center matches the Fermi level. Consequently, the $j-V$ characteristics present negative differential resistance (NDR).

In actual samples, however, the situation is much more complex than this simple picture. This is so even in good-quality heterostructures, when scattering by dislocations or surface roughness is negligible. In particular, inelastic scattering is always present in real devices. An example of inelastic scattering events is electron-electron interaction, in which the energy of the tunneling electron changes and the phase memory is lost. The influence of such manybody effects on DBS has recently attracted considerable attention. Even with its rather satisfactory degree of success, many-body calculations have difficulties that, in some cases, may complicate the interpretation of the underlying physical processes. Recently Presilla et al. [3] pointed out the possibility of nonlocal effective nonlinearities due to many-body interactions in electron transport through DBS. Several results have been obtained using this mean field analysis such as quantum chaos [四] and nonlinear oscillations. [5],6] Loosely speaking, this kind of treatment could be regarded as similar to Hartree-Fock and other self-consistent techniques, which substitute many-body interactions by a nonlinear effective potential. It has to be stressed, however, that the nonlocal interaction might not be the most suitable one in many contexts (e.g., when wells are wide) because it does not take into account the spatial variation of the effective potential. In any event what appears to be clear is that RT in nanostructures is intrinsically a (perhaps weakly) non-linear phenomena as have been shown in many different situations, such as the nonlinearity dependence of 
the lock-in voltage with the number of tunneling electrons obtained with a Hartree-Fock approximation by Wang et al. [0]

In this letter we present a model where the nonlinear interaction is driven by a local field instead of the mean-field approach used by Presilla and co-workers. [3] To describe our model we have chosen a DBS under an applied electric field. The thickness of the whole structure is $L$ and the thickness of the well is $d$. The barriers are assumed to be of the same thickness (symmetric case) but as will be evident below this is not a restriction of our approach. The structure is embedded in a highly doped material acting as contact, so that the electric field is applied only in the DBS. We focus on electron states close to the bandgap and thus we can neglect nonparabolicity effects hereafter. Then the one-band effective-mass framework is completely justified to get accurate results. For the sake of simplicity, we will further assume that the electron effective-mass $m^{*}$ is constant through the whole structure. This hypothesis is related to the fact that for the time being, we are not interested in high quantitative accuracy, although we note that the spatial dependence of the effective mass can be taken into account if necessary.

The generalized effective-mass equation is given by [we use units such that energies are measured in effective Rydberg $\left(\mathrm{Ry}^{*}\right)$ and lengths in effective Bohr radius (a*), being $1 \mathrm{Ry}^{*}=5.5 \mathrm{meV}$ and $1 \mathrm{a}^{*}=100 \AA$ in GaAs]

$$
-\psi_{z z}(z)+\left[V_{E f f}(z)-E\right] \psi(z)=0
$$

where $V_{E f f}(z)$ is the potential term which we discuss below. The DBS can be regarded as an effective medium which reacts to the presence of the tunneling electron, leading to a feedback mechanism by which inelastic scattering processes change the RT characteristics of the device. It thus follows that $V_{E f f}(z)$ must contain nonlinear terms if it is to summarize the medium reaction which comes from the electron-electron and electron-lattice interactions. This term will be a generalized functional of the electronic probability density $\mathcal{F}\left(|\psi(z)|^{2}\right)$. We can now expand this functional as power series in $|\psi(z)|^{2}$, and neglecting higher order (which implies an assumption of weak nonlinearity or weak electron-electron scattering) 
contributions we postulate that the potential in Eq. (1) has the form

$$
V_{E f f}(z)=V_{0}\left[\chi(z)+\tilde{\alpha}|\psi(z)|^{2}\right]-e F z,
$$

where $V_{0}$ is the conduction band-offset at the interfaces, $F$ is the electric field applied along the growth direction, and $\chi(z)$ is the characteristic function of the barriers,

$$
\chi(z)=\left\{\begin{array}{l}
1, \text { if } 0<z<(L-d) / 2, \\
1, \text { if }(L+d) / 2<z<L, \\
0, \text { otherwise, }
\end{array}\right.
$$

and all the nonlinear physics is contained in the coefficient $\tilde{\alpha}$ which we discuss below.

There are two factors that configure the medium nonlinear response to the tunneling electron. First, it goes without saying that there are repulsive electron-electron Coulomb interactions, which should enter the effective potential with a positive nonlinearity, i.e., the energy is increased by local charge accumulations, leading to a positive sign for $\tilde{\alpha}$. Therefore intuitively, a negative sign for $\tilde{\alpha}$ does not appear to be realistic. Nevertheless, we consider also that situation because in some materials, like polar semiconductors, the electron polarizes the surrounding medium creating a local, positive charge density. Hence the electron reacts to this polarization and experiences an attractive potential. This happens, for instance, in the polaron problem in the weak coupling limit, which becomes valid in most semiconductors, and where it can be seen that the lowest band energy state decreases. [8]

We now work starting from Eq. (1) with the definition in Eq. (2) to cast our equations in a more tractable form. For simplicity, and because we are interested in intrinsic DBS features, we consider that the contacts in which the structure is embedded behave linearly. Therefore, the solution of Eq. (11) is a linear combination of traveling waves. As usual in scattering problems, we assume an electron incident from the left and define the reflection, $r$, and transmission, $t$, amplitudes by the relationships

$$
\psi(z)= \begin{cases}A\left(e^{i k_{0} z}+r e^{-i k_{0} z}\right) & z<0, \\ A t e^{i k_{L} z} & z>L,\end{cases}
$$


where $k_{0}^{2}=E, k_{L}^{2}=E+e F L$, and $A$ is the incident wave amplitude. Now we define $\psi(z)=\left(A|t| \sqrt{k_{L}}\right) \phi(z), \alpha=\tilde{\alpha} k_{L}|A t|^{2}$. Notice that $\alpha$ depends on the amplitudes of the incoming and outgoing waves, which will be relevant later. Using Eq. (1) we get

$$
-\phi_{z z}(z)+\left[V_{E f f}(z)-E\right] \phi(z)=0
$$

where now (2) read as

$$
V_{E f f}(z)=V_{0}\left[\chi(z)+\alpha|\phi(z)|^{2}\right]-e F z
$$

To solve the scattering problem in the DBS we develop a similar approach to that given in Ref. [9]. Since $\phi(z)$ is a complex function, we write $\phi(z)=q(z) \exp [i \gamma(z)]$, where $q(z)$ and $\gamma(z)$ are real functions. Inserting this factorization in Eq. (5) we have $\gamma_{z}(z)=q^{-2}(z)$ and

$$
\begin{aligned}
-q_{z z}(z)+\frac{1}{q^{3}(z)}+\left[V_{0} \chi(z)-e F z-E\right] q(z) & + \\
+V_{0} \alpha q^{3}(z) & =0 .
\end{aligned}
$$

This nonlinear differential equation must be supplemented by appropriate boundary conditions. However, using Eq. (4) this problem can be converted into a initial conditions equation. In fact, it is straightforward to prove that

$$
q(L)=k_{L}^{-1 / 2}, q_{z}(L)=0
$$

and that the transmission coefficient is given by

$$
\tau=\frac{4 k_{0} q^{2}(0)}{1+2 k_{0} q^{2}(0)+k_{0}^{2} q^{4}(0)+q^{2}(0) q_{z}^{2}(0)}
$$

Hence, we can integrate numerically (7) with initial conditions (8) backwards, from $z=L$ up to $z=0$, to obtain $q(0)$ and $q_{z}(0)$, thus computing the transmission coefficient for given nonlinear coupling $\alpha$, incoming energy $E$ and applied voltage $V=F L$.

Once the transmission coefficient has been computed, and recalling that contacts are linear media, the tunneling current density at a given temperature $T$ for the DBS can be calculated within the stationary-state model from 


$$
j(V)=\frac{m^{*} e k_{B} T}{2 \pi^{2} \hbar^{3}} \int_{0}^{\infty} \tau(E, V) N(E, V) d E,
$$

where $N(E, V)$ accounts for the occupation of states to both sides of the device, according to the Fermi distribution function, and it is given by

$$
N(E, V)=\ln \left(\frac{1+\exp \left[\left(E_{F}-E\right) / k_{B} T\right]}{1+\exp \left[\left(E_{F}-E-e V\right) / k_{B} T\right]}\right),
$$

where $k_{B}$ is the Boltzmann constant.

In our calculations we have considered a $\mathrm{GaAs}-\mathrm{Ga}_{0.65} \mathrm{Al}_{0.35} \mathrm{As}$ double-barrier structure with $L=3 d=150 \AA$. The conduction-band offset is $V_{0}=250 \mathrm{meV}$. In the absence of applied electric field and nonlinearities, there exists a single, very narrow resonance with $\tau \sim 1$ below the top of the barrier, with an energy of $\sim 81 \mathrm{meV}$, and hence the well supports a single quasi-bound state. Figure 1 shows the transmission coefficient as a function of the incoming energy for different values of the nonlinear coupling $\alpha$ (a) 0 and $10^{-4}$, (b) $10^{-3}$ and (c) $10^{-2}$, at zero bias. Insets show the effective potential $V_{E f f}$ at the energy marked with an arrow. It is clear that the resonances are shifted to energies higher than in the noninteracting case. The shift is produced by the accumulation of charge in the well as shown in the figures of $V_{E f f}$. These results are in very good agreement with self-consistent calculations. [10,[1] Note that $V_{E f f}$ reproduce the charge accumulation in the barriers, close to the heterojunctions, and in the center of the well, obtained in the Hartree approximation. 12] For completeness, we consider also the case when lattice lattice polarization effects are stronger than electron-electron interactions, thus leading to a negative $\alpha$. This regime may give rise to some kind of unphysical "superconducting instability" but for small values of the nonlinear coefficients we have never found that problem. Figure 2 shows the transmission coefficient as a function of the incoming energy for different values of the nonlinear coupling $\alpha$ (a) 0 and $-10^{-4}$, (b) $-10^{-3}$ and (c) $-10^{-2}$, at zero bias. Now the resonances are shifted to lower energies. Also we show that for some values of the nonlinear coefficient there appears another quasi-bound state for lower energies. Looking at the effective potential we conclude that the polarization of the lattice produce a deeper well and consequently the possibility of new bound states, thus shifting or even doubling the original resonance. 
To apply our calculations to obtain measurable quantities, we now discuss the $j-V$ characteristics, as computed from (10). When voltage is applied, the energy of the quasibound state level is lowered and a strong enhancement of the current arises whenever the Fermi level matches this resonance, thus leading to the well-known RT phenomenon. Typical results are shown in Fig. 3 for the particular values (a) $\alpha=10^{-3}$ and (b) $-10^{-3}$. The temperature was $77 \mathrm{~K}$ and Fermi energy was (a) $E_{F}=83 \mathrm{meV}$ and (b) $3 \mathrm{meV}$. In both cases we see clear NDR signatures. For the repulsive interaction we have a NDR peak shifted to higher energies (note the large Fermi energy) than in the linear case as expected in view of Fig 1. The attractive self-interaction causes the occurrence of a second peak at a lower voltage clearly related to the sideband in the transmission coefficient [Fig Q(b)]. A study of bistability [6] may be carried out whitin our model; this kind of results will be obtained in a complete dynamical study of the problem that is in progress.

We have demonstrated that the effective nonlinear interaction we have introduced captures the essential physics of some inelastic effects in RT in a very simple way. The virtue of such an approach is that it allows to gain insight on the features of DBS without the burden of intensive computations providing, in an inexpensive way, a qualitative picture of what is to be expected in particular devices. Our model introduces a local field interaction that reproduce the effective potential in a more realistic way than mean-field approximation proposed in Ref. [3]. Our model can be easily modified to study other heterostructures in an electric field, like superlattices, and including different kinds of impurities and disorder. An important conclusion we draw from this work is that comparison of the result of current measurements with theoretical expectations allows to conclude which effect or group effects (electron-electron interaction or lattice polarization) is responsible for deviations from linearity in any specific material, by just looking at the shifting of the peaks or the appearance of new peaks. Also, it has to be stressed that a mere quantitative comparison between the present work and our idea of effective potentials to Hartree results on the same structure is needed, and we plan to elaborate further on this point in the new future. In addition to realize the study of the interplay between disorder and nonlinearity in this kind of models will 
be very interesting because of the possibility of finding delocalization, driven by nonlinear effects. 13

We are very thankful to Paco Padilla and Alan R. Bishop for useful discussions. This work is supported from CICYT (Spain) through project No. MAT95-0325. 


\section{REFERENCES}

[1] T. C. L. G. Sollner, W. D. Goodhue, P. E. Tannenwald, C. D. Parker, and D. D. Peck, Appl. Phys. Lett. 43, 588 (1984).

[2] B. Ricco and M. Ya. Azbel, Phys. Rev. B 29, 1970 (1984).

[3] C. Presilla, G. Jona-Lasinio, and F. Capasso, Phys. Rev. B 43, 5200 (1991)

[4] G. Jona-Lasinio, C. Presilla, and F. Capasso, Phys. Rev. Lett. 68, 2269 (1992).

[5] G. Jona-Lasinio, C. Presilla, and J. Sjöstrand, Annals of Physics 240, 1 (1995).

[6] N. G. Sun and G. P. Tsironis, Phys. Rev. B 51, 11221 (1995).

[7] L. Wang, J. K. Zhang and A. R. Bishop, Phys. Rev. Lett. 74, 4710 (1995).

[8] J. Callaway, Quantum Theory of the Solid State (Academic Press, CA, 1991), p 711.

[9] R. Knapp, G. Papanicolaou, and B. White, J. Stat. Phys. 63, 567 (1991).

[10] E. Cota and S. E. Ulloa, Phys. Rev. B 51, 10875 (1995).

[11] M. S. Sherwin, K. Craig, B. Galdrikian, J. Heyman, A. Markelz, K. Campman, S. Fafard, P. F. Hopkins, and A. Gossard, Physica D 83, 229 (1995).

[12] G. Bastard, Wave mechanics applied to semiconductor heterostructures (Les Editions de Physique, France, 1988), p 161.

[13] A. Sánchez and L. Vázquez, Int. J. Mod. Phys. B 5, 2825 (1991); S. A. Gredeskul and Yu. S. Kivshar, Phys. Rep. 216, 1 (1992); E. Diez, F. Domínguez-Adame, and A. Sánchez, Phys. Lett. A 198, 403 (1995). 


\section{FIGURES}

FIG. 1. Transmission coefficient $\tau$ as a function of the electron energy at zero bias for (a) $\alpha=10^{-4}$, (b) $10^{-3}$, and (c) $10^{-2}$. For comparison, dashed line indicates in (a) the result for $\alpha=0$. Insets show the effective potential $V_{E f f}$ at the energy marked with an arrow.

FIG. 2. Transmission coefficient $\tau$ as a function of the electron energy at zero bias for (a) $\alpha=-10^{-4}$, (b) $-10^{-3}$, and (c) $-10^{-2}$. For comparison, dashed line indicates in (a) the result for

$\alpha=0$. Insets show the effective potential $V_{E f f}$ at the energy marked with an arrow. Left inset in (c) shows an enlarged view of the lower resonant peak.

FIG. 3. Computed $j-V$ characteristics for $T=77 K$, with (a) $\alpha=10^{-3}, E_{F}=83 \mathrm{meV}$ and (b) $\alpha=-10^{-3}, E_{F}=3 \mathrm{meV}$. 


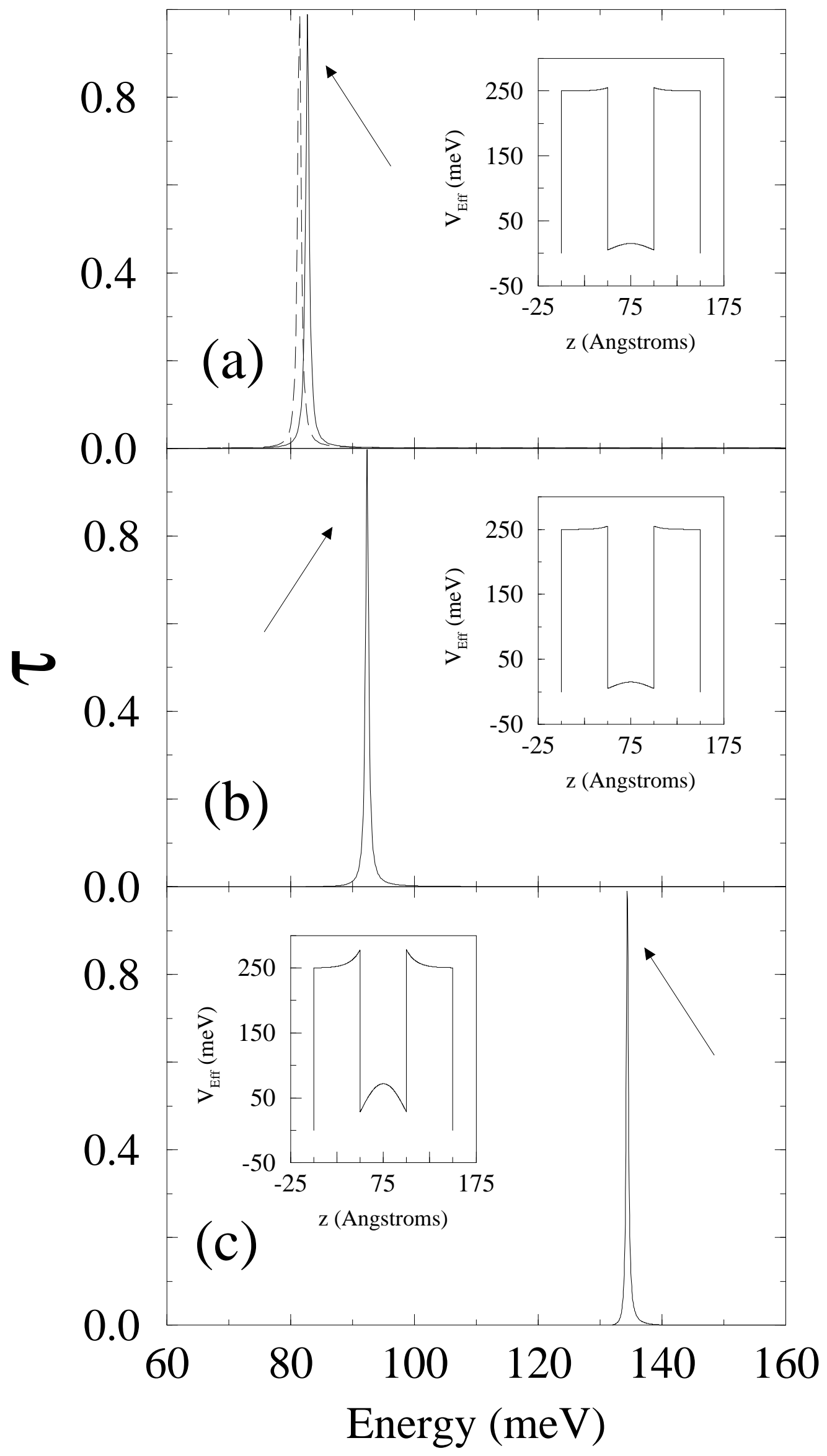




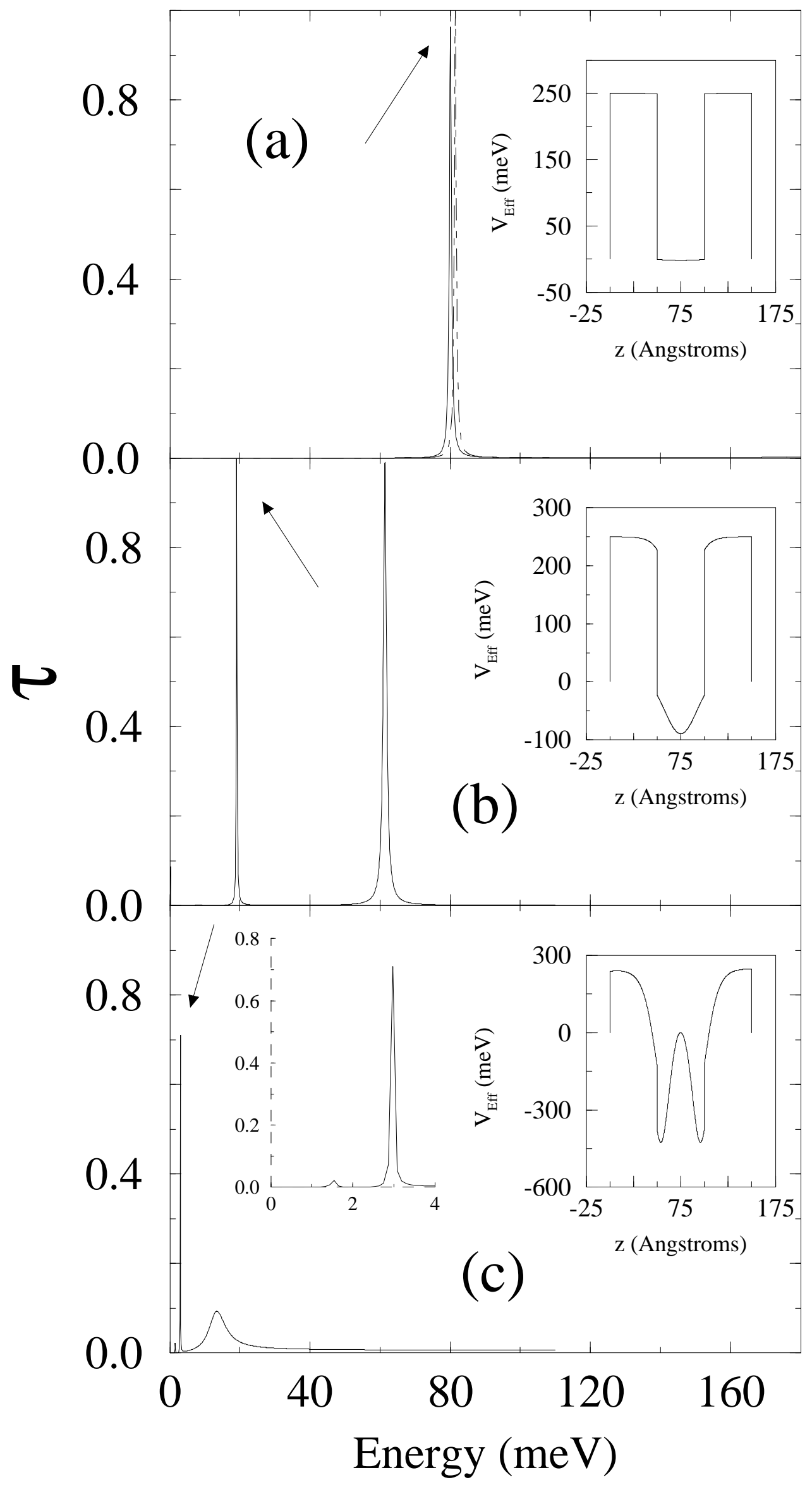




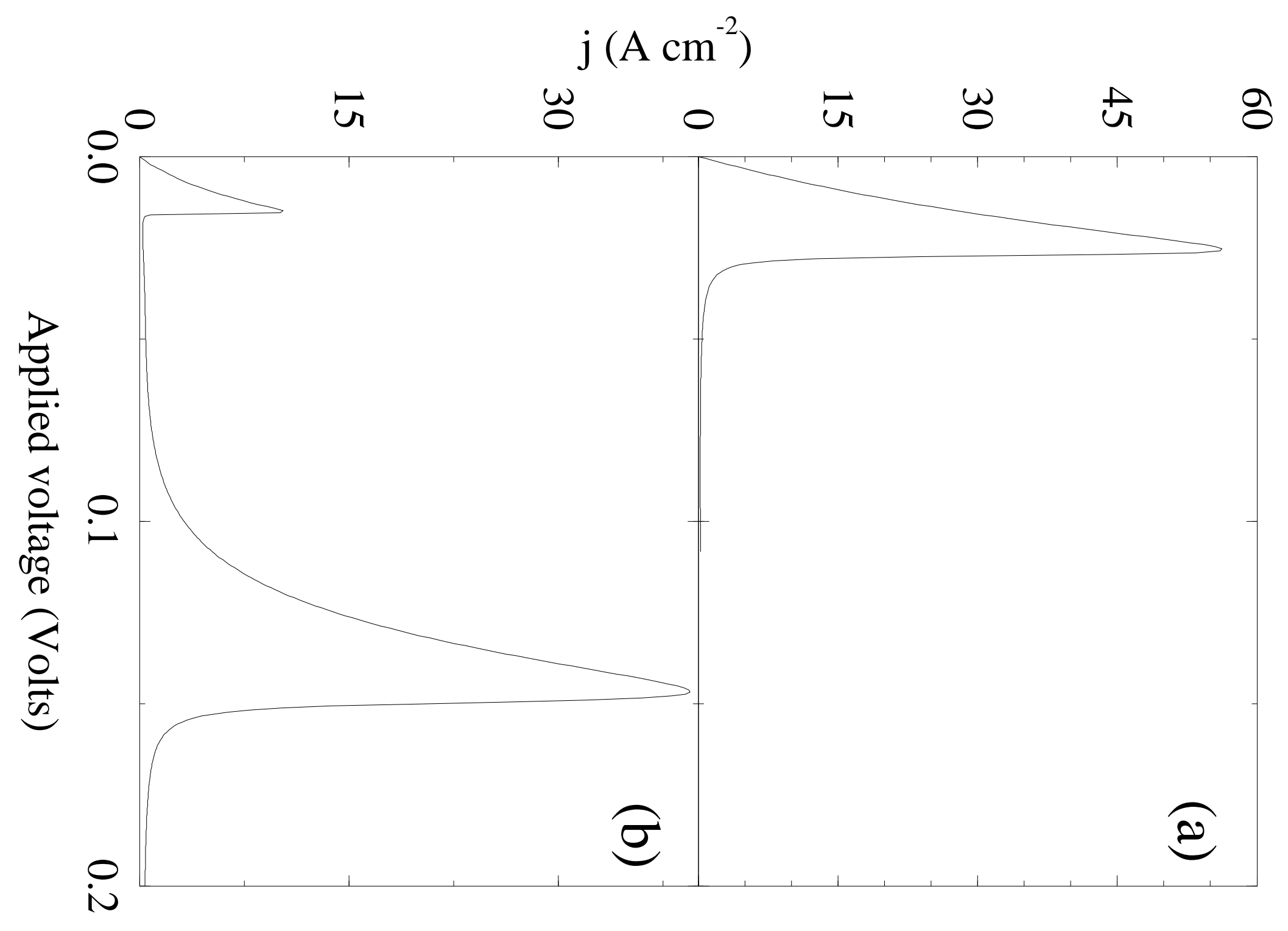

